\title{
Feedback Stabilization Over Signal-to-Noise Ratio Constrained Channels
}

\author{
Julio H. Braslavsky, Member, IEEE, Richard H. Middleton, Fellow, IEEE, and James S. Freudenberg, Fellow, IEEE
}

\begin{abstract}
There has recently been significant interest in feedback stabilization problems with communication constraints including constraints on the available data rate. Signal-to-noise ratio (SNR) constraints are one way in which data-rate limits arise, and are the focus of this paper. In both continuous and discrete-time settings, we show that there are limitations on the ability to stabilize an unstable plant over a SNR constrained channel using finite-dimensional linear time invariant (LTI) feedback. In the case of state feedback, or output feedback with a delay-free, minimum phase plant, these limitations in fact match precisely those that might have been inferred by considering the associated ideal Shannon capacity data rate over the same channel. In the case of LTI output feedback, additional limitations are shown to apply if the plant is nonminimum phase. In this case, we show that for a continuous-time nonminimum phase plant, a periodic linear time varying feedback scheme with fast sampling may be used to recover the original SNR requirement at the cost of robustness properties. The proposed framework inherently captures channel noise effects in a simple formulation suited to conventional LTI control performance and robustness analysis, and has potential to handle time delays and bandwidth constraints in a variety of control over communication links problems.
\end{abstract}

Index Terms-Communication channels, control systems, feedback communication, information rates, linear-quadraticGaussian control, networked control systems, signal-to-noise ratio (SNR).

\section{INTRODUCTION}

$\mathbf{T}$ HE rapid increase in communication and networking technology has prompted many researchers to study limitations imposed upon a feedback control system by the presence of a communication channel in the feedback loop (cf. the papers in [1], [2]-[6], and references therein). There are many types of limitation that such a channel may impose, including quantization, delay, noise, data loss, and bandwidth constraints. A unified treatment of these issues is yet unavailable, and researchers, thus, focus on simplified channel models that highlight certain aspects of the overall problem. For example, several papers have considered an error-free digital channel that imposes a limit on the rate at which quantized data can be transmitted. Using such a channel model, Nair and Evans [3], [7] have derived a tight

Manuscript received March 1, 2006; revised December 21, 2006. Recommended by Associate Editor S. Dey.

J. H. Braslavsky and R. H. Middleton are with the Centre for Complex Dynamic Systems and Control, The University of Newcastle, Callaghan NSW 2308, Australia (e-mail: Julio.Braslavsky@ newcastle.edu.au; Rick.Middleton@newcastle.edu.au).

J. S. Freudenberg is with the Department of Electrical Engineering and Computer Science, University of Michigan, Ann Arbor, MI 48109 USA (e-mail: jfr@eecs.umich.edu).

Digital Object Identifier 10.1109/TAC.2007.902739

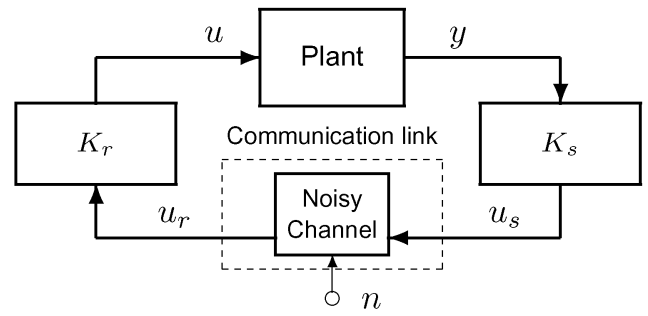

Fig. 1. Feedback control over a communication link.

lower bound on the data rate required to stabilize an unstable discrete-time plant. Similar results have been obtained in [4] and [2], and have also been extended to channels that admit a small, but non-zero, transmission error rate [5].

The present paper considers a control system with feedback over a communication link as shown in Fig. 1. Here, the block $K_{s}$ encapsulates processes such as encoding, sensor filtering, and control computations to generate the signal $u_{s}$ sent to the communication channel, while the block $K_{r}$ encapsulates processes such as decoding and actuator signal processing based on the received signal $u_{r}$. The plant is assumed to be a finite-dimensional (FD) linear time invariant (LTI) system.

The aforementioned results for data-rate limited channels typically include two components: necessary conditions for stabilization based on information theoretic arguments, and complex, nonlinear, time varying, encoding and decoding algorithms that show sufficiency of the data-rate for stabilization. The sufficiency component of these results is not claimed to possess any particular robustness properties (e.g., is "not intended ... to be a practical solution" [3, p. 431]), and is quite disparate from modern control performance and robustness analysis techniques.

To resolve this disparity, in the present paper we frame our approach to feedback under communication constraints with the following two core postulates.

1) We adopt a signal-to-noise ratio (SNR) constraint model of communication capacity limits.

2) We restrict our attention to finite dimensional, linear feedback. Namely, the blocks $K_{s}$ and $K_{r}$ in Fig. 1 consist at most of linear filtering and sample and hold operations.

The proposed SNR constraint model of communication capacity limits is motivated by the observation that properties of an additive white Gaussian noise (AWGN) channel with an input power constraint may be used to derive a limit on the channel data rate consistent with reliable communication. Indeed, following [8]-[11], denote the available transmission power by $\mathcal{P}$ $\mathrm{W}$, the noise power by $\mathcal{N} \mathrm{W}$, and the channel bandwidth by $W$ $\mathrm{Hz}$. Then, the channel capacity is given by the famous formula 
$\mathcal{C}=W \log _{2}(1+\mathcal{P} / \mathcal{N})$ bits/s. The data rate limit is, thus, determined by the channel bandwidth $W$ and SNR $\mathcal{P} / \mathcal{N}$. Suppose further that the noise has spectral density $N_{0} / 2 \mathrm{~W} / \mathrm{Hz}$. Then, the noise power is given by $\mathcal{N}=N_{0} W \mathrm{~W}$, and taking the limit as $W \rightarrow \infty$ yields $\mathcal{C}=\left(\log _{2} e\right) \mathcal{P} / N_{0}$ bits/s [10], [11]. Hence, with infinite bandwidth, the channel capacity is determined by the ratio of transmission power to noise spectral density, $\mathcal{P} /\left(N_{0} / 2\right) \mathrm{Hz}$. By a mild abuse of terminology, we shall also refer to this quantity as the SNR.

There are several reasons behind the postulate of linearity in our approach. On the one hand, by restricting our attention to linear elements, we develop a framework for the analysis of feedback under communication constraints that naturally incorporates a large number of well-established performance and robustness tools. On the other hand, there are reasons of specific significance to feedback stabilization under data-rate constraints.

- First, for LTI or periodically linear time varying (LTV) elements $K_{s}$ and $K_{r}$, the resulting stability of the loop in Fig. 1 is exponential, which, as is well known, guarantees a nonvanishing robustness margin. As a consequence, our bounds on required SNR for stability inherently account for a degree of robustness.

- Second, for minimum phase, delay-free plants, we show that the consideration of a more general class of causal nonlinear time-varying elements, as in [3]-[5], [7], yields the same requirement on channel capacity obtained by considering LTI elements only.

- Finally, for plants with nonminimum phase zeros, or time delays, we show that LTI solutions will necessarily demand additional communication capacity, as would be expected in practice due to robustness and other considerations [12]-[15] [2, Remark 1].

Our results, and the body of the paper, are outlined as follows. We first consider the problem of stabilizing a continuous-time unstable plant with an analog communication channel in the feedback loop. In Section II-A, we assume that the states of the plant are available for feedback, and derive the minimal SNR compatible with stabilization using linear state feedback. To compare our result with that of Nair and Evans, we compute the channel capacity corresponding to this SNR using the formula for an infinite bandwidth AWGN channel. The resulting capacity is equal, in units of bits/s, to the minimal data rate derived by Nair and Evans [3], [7]. In Section II-B, we assume that only the plant output is available for feedback, and show that if the plant is both unstable and nonminimum phase, then the minimal SNR required for stabilization by an LTI controller is strictly greater than that in the state feedback case. As a consequence, the capacity associated with the minimal SNR is greater than the Nair and Evans data rate. Since our results assume a linear time-invariant control law, it is natural to ask whether time-varying control can achieve stabilization with a lower SNR. To pursue this question, we first discretize the plant and channel using appropriately chosen sample and hold functions, and then design a discrete-time controller using the results of Section III. In this section, we compute the minimal SNR compatible with stabilizing a discrete-time plant over a discrete-time noisy channel, and show that the resulting dis- crete channel capacity is consistent with the Nair and Evans data rate only if the plant is minimum phase and has relative degree one. Since relative degree in discrete-time implies delay in the system response, our discrete-time results show that stabilization with delay will necessarily require a larger SNR. ${ }^{1}$ In Section IV we apply the results of Section III to obtain a linear, periodically time varying control law that stabilizes a nonminimum phase analog plant with a SNR arbitrarily close to that achievable with state feedback. However, such LTV control laws can achieve significant reduction in SNR only at the expense of stability robustness. In a similar vein, we show that as in the data-rate stabilization case [5], [17], asymptotic stability can be achieved using an LTV compensator. However, a consequence of this approach is extreme sensitivity to unmodeled noise and disturbances. The paper is summarized and future research directions are described in Section V.

Preliminary versions of the results in the present paper have been communicated in [18] and [19].

Terminology: Denote by $\mathbb{C}^{-}, \overline{\mathbb{C}}^{-}, \mathbb{C}^{+}$and $\overline{\mathbb{C}}^{+}$, respectively the open-left, closed-left, open-right, and closed-right halves of the complex plane $\mathbb{C}$. The open unit disk is denoted $\mathbb{D}$, the closed unit disk $\overline{\mathbb{D}}$, and their complements by $\mathbb{D}^{\mathrm{C}}$ and $\overline{\mathbb{D}}^{\mathrm{C}}$, respectively. A continuous-time signal is denoted by $x(t), t \in \mathbb{R}$, and its Laplace transform by $X(s), s \in \mathbb{C}$. A discrete-time signal is denoted by $x_{k}, k \in \mathbb{Z}$, and correspondingly its $Z$ transform by $X(z), z \in \mathbb{C}$. Whenever the arguments of $X(s)$ or $X(z)$ are omitted, the meaning will be clear from the context. A square matrix $A \in \mathbb{R}^{n \times n}$ is called Hurwitz if all its eigenvalues are in $\mathbb{C}^{-}$; it is called Schur if all its eigenvalues are in $\mathbb{D}$. The expectation operator is denoted by $\mathrm{E}$. A rational transfer function of a continuous-time system is termed minimum phase if all its zeros lie in $\overline{\mathbb{C}}^{-}$, and is nonminimum phase if it has zeros in $\mathbb{C}^{+}$. Similarly, a discrete-time transfer function is minimum phase if all its zeros lie in $\overline{\mathbb{D}}$ and NMP otherwise. Given $G(s)$, the transfer function of a continuous-time system, we say that $G(s) \in \mathrm{H}_{2}$ if $G(s)$ is strictly proper and stable; i.e., all its poles lie in $\mathbb{C}^{-}$. The $H_{2}$ norm of $G(s)$, denoted by $\|G\|_{H_{2}}$, satisfies $\|G\|_{H_{2}}^{2}=(1 / 2 \pi) \int_{-\infty}^{\infty}|G(j \omega)|^{2} d \omega$. Similarly, given $G(z)$, the transfer function of a discrete-time system, we say that $G(z) \in H_{2}(\mathbb{D})$ if $G(z)$ is strictly proper and stable; i.e., all its poles lie in $\mathbb{D}$. The $H_{2}(\mathbb{D})$ norm of $G(z)$, denoted by $\|G\|_{H_{2}(\mathbb{D})}$, satisfies $\|G\|_{H_{2}(\mathbb{D})}^{2}=(1 / 2 \pi) \int_{-\pi}^{\pi}\left|G\left(e^{j \theta}\right)\right|^{2} d \theta$. In the sequel, we shall use the notation $\|G\|_{H_{2}}$ to denote both norms, as the meaning will be clear from the context.

\section{Continuous-Time FeEdBack Channels}

We now consider the problem of stabilizing an unstable continuous-time plant by using feedback over a noisy continuoustime communication channel. Let the plant have transfer function $G(s)$ and state equations ${ }^{2}$

$$
\dot{x}(t)=A x(t)+B u_{r}(t), \quad y(t)=C x(t)
$$

${ }^{1}$ Computing the minimal SNR for continuous-time systems with delay is technically more difficult, and is addressed in [16].

${ }^{2} \mathrm{~A}$ mathematically precise treatment of continuous-time stochastic systems requires the theory of stochastic differential equations. However, under reasonable assumptions, that formulation reduces to the one presented here [20, §4.4]. 
where $x \in \mathbb{R}^{n}, u_{r} \in \mathbb{R}, y \in \mathbb{R}$, and the triple $(A, B, C)$ is assumed minimal. We assume an infinite bandwidth AWGN channel with input output relation

$$
u_{r}(t)=u_{s}(t)+n(t), \quad t \geq 0, t \in \mathbb{R}
$$

where $u_{s}(t)$ is the channel input, or "sent" signal, $u_{r}(t)$ is the channel output, or "received" signal, and $n(t)$ is zero-mean white Gaussian noise with power spectral density ${ }^{3} \Phi$. We restrict attention to the case where the overall feedback system is stabilized, such that for any distribution of initial conditions, the distribution of all signals converges exponentially rapidly to a stationary distribution. Without loss of generality, we, therefore, consider directly the properties of the stationary distribution of the relevant signals. Denote the power spectral density of $u_{s}(t)$ by $S_{u_{s}}(\omega)$. The power in the channel input, defined by $\left\|u_{s}\right\|_{\text {Pow }} \stackrel{\triangleq}{\triangleq} \mathrm{E}\left\{u_{s}^{2}(t)\right\}$, is related to its spectral density by

$$
\left\|u_{s}\right\|_{\text {Pow }}=\frac{1}{2 \pi} \int_{-\infty}^{\infty} S_{u_{s}}(\omega) d \omega .
$$

The channel input is required to satisfy the power constraint

$$
\left\|u_{s}\right\|_{\text {Pow }}<\mathcal{P}
$$

for some predetermined input power level $\mathcal{P}>0$. A power constraint such as (4) may arise either from electronic hardware limitations or regulatory constraints introduced to minimize interference to other communication system users. The infinite bandwidth AWGN channel is, thus, characterized by two parameters: the admissible input power level $\mathcal{P}$, and the noise spectral density $\Phi$. As we have noted in Section I, channel capacity depends solely upon the SNR $\mathcal{P} / \Phi$ radians/second.

\section{A. Continuous-Time State Feedback Stabilization}

In this section, we consider the feedback system of Fig. 2 in which the channel input is static state feedback $u_{s}(t)=$ $-K x(t)$. This is a special case of Fig. 1 with $K_{r}=1$ and $K_{s}=-K$. It will be clear from the development that there is no loss of generality in using only compensation at the channel input, and in assuming a static feedback law. The closed loop transfer function from channel noise $n(t)$ to channel input $u_{s}(t)$ is equal to $-T^{s f}(s)$, where

$$
T^{s f}(s)=\frac{K(s I-A)^{-1} B}{1+K(s I-A)^{-1} B}
$$

is the complementary sensitivity function of the state feedback system. If the closed loop system is stable, then $u_{s}(t)$ is a stationary Gaussian stochastic process with power spectral density $S_{u_{s}}(\omega)=\left|T^{s f}(j \omega)\right|^{2} \Phi$, and, thus, the power of the channel input signal is related to the $H_{2}$ norm of $T_{s f}$

$$
\left\|u_{s}\right\|_{\mathrm{Pow}}=\left\|T^{s f}\right\|_{H_{2}}^{2} \Phi .
$$

${ }^{3}$ In contrast with standard notation [8]-[11], we denote the noise density by $\Phi \mathrm{W} /\left(\right.$ radians/s) instead of $N_{0} / 2 \mathrm{~W} / \mathrm{Hz}$.

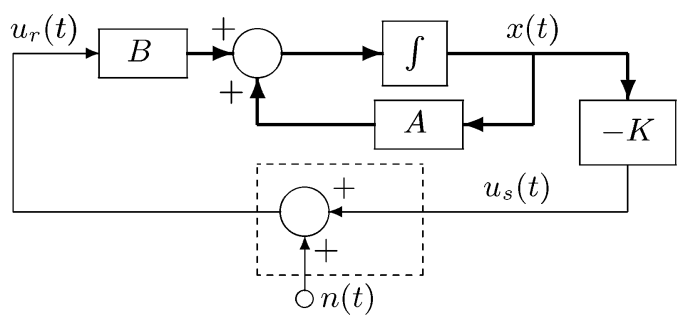

Fig. 2. State feedback stabilization of an analog system over an AWGN channel.

It follows that the input power constraint (4) is equivalent to a bound imposed on the complementary sensitivity function by the admissible channel SNR:

$$
\left\|T^{s f}\right\|_{H_{2}}^{2}<\frac{\mathcal{P}}{\Phi} .
$$

Denote the class of all stabilizing state feedback gains by $\mathcal{K}_{s f}=$ $\{K: A-B K$ is Hurwitz $\}$.

Problem 1 (SNR Constrained State Feedback Stabilization): Find a static state feedback gain $K \in \mathcal{K}_{s f}$ such that the complementary sensitivity function (5) satisfies the constraint (6) imposed by the admissible channel SNR.

Theorem II.1: Consider the feedback system of Fig. 2, suppose that $A$ has $\mathbb{C}^{+}$eigenvalues $\left\{p_{i} ; i=1,2, \ldots, m\right\}$ and define $T^{s f}(s)$ as in (5). Then

$$
\inf _{K \in \mathcal{K}_{s f}}\left\|T^{s f}\right\|_{H_{2}}^{2}=2 \sum_{i=1}^{m} \operatorname{Re}\left\{p_{i}\right\}
$$

and Problem 1 is solvable if and only if the admissible SNR satisfies

$$
\frac{\mathcal{P}}{\Phi}>2 \sum_{i=1}^{m} \operatorname{Re}\left\{p_{i}\right\} \quad \text { radians/s. }
$$

Proof: See Appendix A, which also contains a procedure for finding the desired gain.

It follows from (8) that stabilization requires an AWGN channel whose capacity satisfies the lower bound

$$
\mathcal{C}=\left(\log _{2} e\right) \mathcal{P} / 2 \Phi>\left(\log _{2} e\right) \sum_{i=1}^{m} \operatorname{Re}\left\{p_{i}\right\} \quad \text { bits/s. }
$$

Let us now compare our results to those of Nair and Evans [7], who consider the problem of using output feedback to stabilize the discrete-time system

$$
x_{k+1}=A_{d} x_{k}+B_{d} u_{k}, \quad y_{k}=C_{d} x_{k}
$$

where $\forall k=0,1,2, \ldots, A_{d}$ has $\overline{\mathbb{D}}^{\mathrm{C}}$ eigenvalues $\left\{\phi_{i} ; i=\right.$ $1, \ldots, m\}$, and the initial state is a random variable. Nair and Evans show that exponential stabilization over an error-free digital channel is possible if and only if the data rate $R$ satisfies

$$
R>\sum_{i=1}^{m} \log _{2}\left|\phi_{i}\right| \quad \text { bits/transmission. }
$$




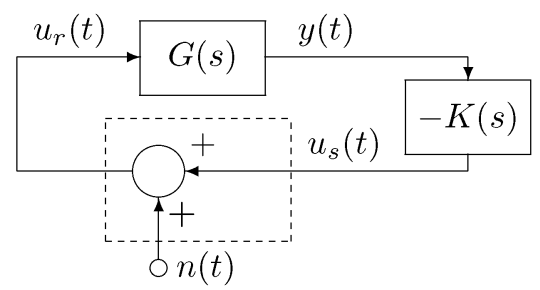

Fig. 3. Stabilization of an analog system via output feedback over an AWGN channel.

Using the concept of topological feedback entropy, the authors of [21] show that the right-hand side (RHS) of (11) is a measure of the rate at which the open loop plant generates information about its initial state.

To compare with our results, suppose that the discrete-time system (10) is obtained by sampling the continuous-time system (1) at a constant rate $1 / T \mathrm{~Hz}$. Then the $\overline{\mathbb{D}}^{\mathrm{C}}$ eigenvalues of $A_{d}$ must satisfy $\phi_{i}=e^{p_{i} T}$, where $\left\{p_{i}\right\}$ are the $\mathbb{C}^{+}$eigenvalues of $A$, and (11) reduces to

$$
R / T>\left(\log _{2} e\right) \sum_{i=1}^{m} \operatorname{Re}\left\{p_{i}\right\} \quad \text { bits/s. }
$$

By comparing (9) to (12), we see that the capacity associated with the SNR required for stabilization over an AWGN channel with state feedback is equal, in units of bits/s, to the data rate required for stabilization over an error-free digital channel with output feedback.

The necessary and sufficient conditions for stabilizability derived by Nair and Evans [3], [7] are obtained using a very general class of feedback control laws assumed only to be causal. To demonstrate sufficiency they construct a complex coding and decoding feedback mechanism [3], [7] (cf. [5]). It is, thus, of interest to the present results to demonstrate sufficiency using a linear time invariant control law.

\section{B. Continuous-Time Output Feedback Stabilization}

We now turn to the feedback system of Fig. 3, in which the channel input is dynamic output feedback: $U_{s}(s)=-K(s) Y(s)$. The closed loop transfer function from channel noise $n(t)$ to channel input $u_{s}(t)$ is equal to $-T(s)$, where $T$ is the complementary sensitivity function of the output feedback loop

$$
T(s)=\frac{G(s) K(s)}{1+G(s) K(s)} .
$$

If the feedback system is stable, then the power of the channel input signal is given by

$$
\left\|u_{s}\right\|_{\text {Pow }}=\|T\|_{H_{2}}^{2} \Phi .
$$

As in the preceding section, we see that the input power constraint (4) may be restated as a constraint imposed on the complementary sensitivity function (13) by the admissible channel SNR, specifically

$$
\|T\|_{H_{2}}^{2}<\frac{\mathcal{P}}{\Phi} .
$$

Let $\mathcal{K}$ denote the class of all proper controllers $K(s)$ that internally stabilize the feedback system of Fig. 3.

Problem 2 (SNR Constrained Output Feedback Stabilization): Find a proper rational function $K(s) \in \mathcal{K}$ such that the complementary sensitivity function (13) satisfies the constraint (14) imposed by the admissible channel SNR.

Denote the Blaschke product containing the $\mathbb{C}^{+}$poles of $G(s)$ by

$$
B_{p}(s)=\prod_{i=1}^{m} \frac{s-p_{i}}{s+\bar{p}_{i}} .
$$

Theorem II.2: Consider the feedback system of Fig. 3, assume that $A$ has $\mathbb{C}^{+}$eigenvalues $\left\{p_{i} ; i=1,2, \ldots, m\right\}$, and define $T$ as in (13). Denote the nonminimum phase zeros of $G(s)$ by $\left\{z_{i} ; i=1, \ldots, q\right\}$, and assume that these zeros are distinct. ${ }^{4}$ Then

$$
\inf _{K(s) \in \mathcal{K}}\|T\|_{H_{2}}^{2}=2 \sum_{i=1}^{m} \operatorname{Re}\left\{p_{i}\right\}+\eta
$$

where

$$
\begin{gathered}
\eta \triangleq \sum_{\ell=1}^{q} \sum_{i=1}^{q}\left(\frac{\gamma_{\ell} \bar{\gamma}_{i}}{\left(z_{\ell}+\bar{z}_{i}\right)}\right) \\
\gamma_{\ell} \triangleq 2 \operatorname{Re}\left\{z_{\ell}\right\}\left(1-B_{p}^{-1}\left(z_{\ell}\right)\right) \prod_{\substack{i=1 \\
i \neq \ell}}^{q} \frac{\left(z_{\ell}+\bar{z}_{i}\right)}{\left(z_{\ell}-z_{i}\right)}
\end{gathered}
$$

and $\eta>0$ whenever $G(s)$ is both unstable and nonminimum phase. It follows that Problem 2 is solvable if and only if the admissible SNR satisfies

$$
\frac{\mathcal{P}}{\Phi}>2 \sum_{i=1}^{m} \operatorname{Re}\left\{p_{i}\right\}+\eta \quad \text { radians/s. }
$$

Proof: See Appendix B, which also includes a derivation of the desired compensator.

If the plant is minimum phase, then (19) implies that the SNR required for stabilization with output feedback is identical to that with state feedback. If the plant is nonminimum phase, then the SNR required for LTI output feedback stabilization is strictly greater than if state feedback is used, and will be particularly large if $G(s)$ has a NMP zero in close proximity to a $\mathbb{C}^{+}$pole. Indeed, in the case of a single (real) NMP zero, the expression (17) for the additional cost due to this zero simplifies to $\eta=$ $2 z\left(1-B_{p}^{-1}(z)\right)^{2}$. If $G(s)$ has but a single real $\mathbb{C}^{+}$pole $p$ and a single real NMP zero $z$, then (19) implies that the required SNR must satisfy the bound

$$
\frac{\mathcal{P}}{\Phi}>2 p\left(\frac{z+p}{z-p}\right)^{2} .
$$

The difficulties imposed upon feedback design by the presence of NMP zeros near to $\mathbb{C}^{+}$poles are well known. Denote the Blaschke product of NMP zeros of $G(s)$ by

$$
B_{z}(s) \triangleq \prod_{i=1}^{q} \frac{s-z_{i}}{s+\bar{z}_{i}}
$$

${ }^{4}$ This assumption is made for simplicity and may be relaxed, as discussed in the proof. 
Then, at each $\mathbb{C}^{+}$pole $p$ of $G(s)$, the complementary sensitivity function must satisfy the bound $[15$, p. 70$]$

$$
\sup _{\omega}|T(j \omega)| \geq\left|B_{z}^{-1}(p)\right|
$$

and will, thus, exhibit a large peak if there is a NMP zero close to $p$. Such a peak implies that the feedback system will possess a small stability margin and exhibit large response to measurement noise [15], and, thus, a larger SNR will be required for LTI output feedback stabilization. We shall return to the stability robustness problems associated with approximate unstable pole/zero cancellations at the close of Section IV.

We have seen that the presence of NMP plant zeros implies that the SNR required to stabilize an unstable plant by LTI feedback is strictly greater than if such zeros were not present. By the argument at the close of Section II-A, it follows that the capacity of the associated AWGN channel is also greater than that for a minimum phase plant with the same unstable poles. As a result, using LTI control to stabilize an unstable and nonminimum phase plant over an AWGN channel requires that the channel capacity be strictly greater than the minimal data rate derived by Nair and Evans, which depends only on the unstable poles.

There is no apparent reason why the channel capacity required for output feedback stabilization should be equal to the data rate derived in [3] and [7]. First, the problem statements are different: stabilization over a noise free date rate limited channel versus stabilization over an SNR constrained AWGN channel. Second, the classes of control algorithms are different: in the present paper we have assumed a linear time invariant controller, whereas the authors of [3] and [7] use control laws that are time varying and, because of the quantizer, inherently nonlinear. In fact, we shall show in Section IV that the use of a linear time varying controller allows our results to be reconciled with those of Nair and Evans, at the cost of potential robustness difficulties. To do so requires the use of sampled-data control and, thus, in Section III, we must first derive discrete-time counterparts to Theorems II-1 and II-2.

\section{Discrete-Time FeEdBack ChanNels}

We now turn to the problem of using state or output feedback to stabilize an unstable discrete-time plant over a noisy discrete-time channel, and develop results parallel to those in Section II. Let the plant have transfer function $G_{d}(z)$ and state variable description (10), and assume that $\left(A_{d}, B_{d}, C_{d}\right)$ is minimal. We assume a discrete-time Gaussian channel with input output relation

$$
w_{k}=v_{k}+n_{k}, \quad k=0,1,2, \ldots
$$

where $n_{k}$ is zero mean Gaussian white noise with variance $\sigma^{2}$. The channel input $v_{k}$ is assumed to be a discrete-time stationary stochastic process with power spectral density $S_{v}(\omega)$. The power in the channel input, defined by $\|v\|_{\text {Pow }} \triangleq \mathrm{E}\left\{v_{k}^{2}\right\}$ may be computed from its spectral density by

$$
\|v\|_{\mathrm{Pow}}=\frac{1}{2 \pi} \int_{-\pi}^{\pi} S_{v}(\omega) d \omega .
$$

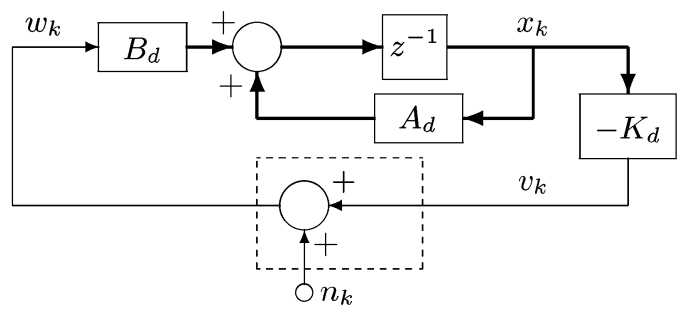

Fig. 4. Stabilization of a discrete-time system via state feedback over a discrete Gaussian channel.

Note that the power in a discrete-time white noise signal is equal to its variance. The discrete channel input is required to satisfy the power constraint

$$
\|v\|_{\text {Pow }}<\mathcal{P}_{d}
$$

for some predetermined input power level $\mathcal{P}_{d}$. The capacity of the discrete Gaussian channel is given by $C=(1 / 2) \log _{2}(1+$ $\left.\mathcal{P}_{d} / \sigma^{2}\right)$ bits/transmission [10], [11], and, thus, we see that the channel capacity depends only on the SNR $\mathcal{P}_{d} / \sigma^{2}$.

\section{A. Discrete-Time State Feedback Stabilization}

Consider the discrete-time state feedback system of Fig. 4, in which the channel input is static state feedback: $v_{k}=-K_{d} x_{k}$. The closed loop transfer function from channel noise $n_{k}$ to channel input $v_{k}$ is given by $-T_{d}^{s f}$, where

$$
T_{d}^{s f}(z)=\frac{K_{d}\left(z I-A_{d}\right)^{-1} B_{d}}{1+K_{d}\left(z I-A_{d}\right)^{-1} B_{d}}
$$

is the complementary sensitivity function of the discrete state feedback loop. If the feedback system is stable, then the channel input is stationary and Gaussian with power spectral density $S_{v}(\omega)=\left|T_{d}^{s f}\left(e^{j \omega}\right)\right|^{2} \sigma^{2}$. The power in the channel input is thus given by $\|v\|_{\text {Pow }}=\left\|T_{d}^{s f}\right\|_{H_{2}}^{2} \sigma^{2}$, and the input power constraint (23) is equivalent to the constraint

$$
\left\|T_{d}^{s f}\right\|_{H_{2}}^{2}<\frac{\mathcal{P}_{d}}{\sigma^{2}}
$$

imposed on $T_{d}^{s f}$ by the admissible channel SNR. Denote the class of all stabilizing state feedback gains by $\mathcal{K}_{d}^{s f}=\left\{K_{d}\right.$ : $\left(A_{d}-B_{d} K_{d}\right)$ is Schur $\}$

Problem 3 (Discrete-Time SNR Constrained State Feedback Stabilization): Find a static state feedback gain $K_{d} \in \mathcal{K}_{d}^{s f}$ such that $T_{d}^{s f}$ satisfies the SNR constraint (25).

Theorem III.1: Consider the feedback system in Fig. 4, assume that $A_{d}$ has $\overline{\mathbb{D}}^{\mathrm{C}}$ eigenvalues $\left\{\phi_{i} ; i=1,2, \ldots, m\right\}$, and define $T_{d}^{s f}(z)$ as in (24). Then

$$
\inf _{K \in \mathcal{K}_{d}^{s f}}\left\|T_{d}^{s f}\right\|_{H_{2}}^{2}=\left(\prod_{i=1}^{m}\left|\phi_{i}\right|^{2}\right)-1
$$

and Problem 3 is solvable if and only if the admissible SNR satisfies

$$
\frac{\mathcal{P}_{d}}{\sigma^{2}}>\left(\prod_{i=1}^{m}\left|\phi_{i}\right|^{2}\right)-1 .
$$




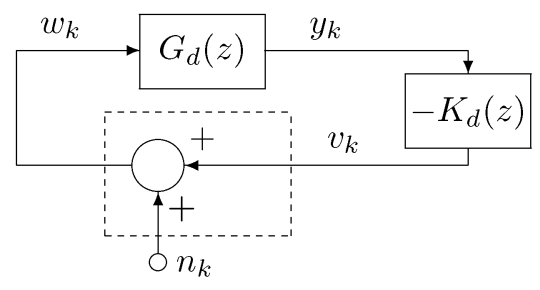

Fig. 5. Stabilization of a discrete-time system via output feedback over a discrete Gaussian channel.

Proof: See Appendix C, which also contains a procedure for finding the desired gain.

It follows from (27) that stabilization via state feedback requires a discrete Gaussian channel whose capacity satisfies

$$
\mathcal{C}=\frac{1}{2} \log _{2}\left(1+\frac{\mathcal{P}_{d}}{\sigma^{2}}\right)>\sum_{i=1}^{m} \log _{2}\left|\phi_{i}\right| \quad \text { bits/transmission }
$$

and is, thus, consistent with the data rate (11) derived by Nair and Evans [7]. As discussed in [22], Shannon capacity is a "tight" channel figure of merit to characterize the equivalence between feedback stability and reliable communication in the case of AWGN channels.

\section{B. Discrete-Time Output Feedback Stabilization}

Consider the discrete-time feedback system of Fig. 5, where the channel input is dynamic output feedback $V(z)=-K_{d}(z) Y(z)$. If the feedback system is stable, then

$$
\|v\|_{\text {Pow }}=\left\|T_{d}\right\|_{H_{2}}^{2} \sigma^{2}
$$

where

$$
T_{d}(z)=\frac{K_{d}(z) G_{d}(z)}{1+K_{d}(z) G_{d}(z)}
$$

is the complementary sensitivity function of the discrete output feedback loop. The input power constraint (23) imposed by the admissible SNR is, thus, equivalent to requiring that $T_{d}$ satisfy the bound

$$
\left\|T_{d}\right\|_{H_{2}}^{2}<\frac{\mathcal{P}_{d}}{\sigma^{2}}
$$

Denote the class of all stabilizing output feedback controllers by $\mathcal{K}_{d}$.

Problem 4 (Discrete-Time SNR Constrained Output Feedback Stabilization): Find a proper rational function $K_{d}(z) \in \mathcal{K}_{d}$ such that the complementary sensitivity function (29) satisfies the constraint (30) imposed by the admissible channel SNR.

Denote the Blaschke product containing the $\overline{\mathbb{D}}^{\mathrm{C}}$ poles of $G_{d}(z)$ by

$$
B_{\phi}(z)=\prod_{i=1}^{m} \frac{z-\phi_{i}}{1-z \bar{\phi}_{i}}
$$

and define

$$
\left.\beta_{k} \triangleq \frac{1}{k !} \frac{d^{k}}{d z^{k}} B_{\phi}(z)\right|_{z=0}
$$

Theorem III.2: Consider the feedback system of Fig. 5, assume that $A_{d}$ has $\overline{\mathbb{D}}^{\mathrm{C}}$ eigenvalues $\left\{\phi_{i} ; i=1,2, \ldots, m\right\}$, and define $T_{d}(z)$ as in (29). Let $G_{d}(z)$ have relative degree $r \geq$ 1 , denote the nonminimum phase zeros of $G_{d}(z)$ by $\left\{\zeta_{i} ; i=\right.$ $1, \ldots, q\}$, and assume that these zeros are distinct. ${ }^{5}$ Then

$$
\inf _{K_{d}(z) \in \mathcal{K}_{d}}\left\|T_{d}\right\|_{H_{2}}^{2}=\left(\prod_{i=1}^{m}\left|\phi_{i}\right|^{2}\right)-1+\eta+\delta
$$

where

$$
\begin{gathered}
\eta=\sum_{\ell=1}^{q} \sum_{i=1}^{q} \frac{\gamma_{\ell} \bar{\gamma}_{i}}{\left(\zeta_{\ell} \bar{\zeta}_{i}-1\right)}, \quad \delta= \begin{cases}0, & \text { if } r=1 \\
\sum_{k=1}^{r-1}\left|\beta_{k}\right|^{2}, & \text { if } r>1\end{cases} \\
\gamma_{\ell} \triangleq\left(1-\left|\zeta_{\ell}\right|^{2}\right)\left(B_{\phi}^{-1}\left(\zeta_{\ell}\right)-\sum_{k=0}^{r-1} \beta_{k} \zeta_{\ell}^{-k}\right) \prod_{\substack{k=1 \\
k \neq \ell}}^{q} \frac{1-\zeta_{\ell} \bar{\zeta}_{k}}{\zeta_{\ell}-\zeta_{k}}
\end{gathered}
$$

and $\eta>0$ unless $G_{d}(z)$ is minimum phase. It follows that Problem 4 is solvable if and only if the admissible SNR satisfies

$$
\frac{\mathcal{P}_{d}}{\sigma^{2}}>\left(\prod_{i=1}^{m}\left|\phi_{i}\right|^{2}\right)-1+\eta+\delta .
$$

Proof: See Appendix D, which also includes a derivation of the desired compensator.

As in the continuous-time case, the SNR required for stabilization of a NMP plant via output feedback is strictly greater than that required for state feedback. The required SNR also depends on the relative degree of the plant, as is reasonable because a discrete-time system with relative degree $r$ possesses an $r$-step delay in its response, and delay is known to aggravate the problem of robust stabilization (cf. the discussion of the discrete Poisson complementary sensitivity integral on $[15, \mathrm{pp} .77$, 78]). A discrete-time system obtained by discretizing a finite dimensional continuous-time system will generally have relative degree one; however, a continuous-time system with a pure time delay will yield a discretized system with higher relative degree [23], [24].

Remark III.1: Suppose that the discrete-time plant has relative degree $r=2$. Then, the additional SNR due to the relative degree is $\delta=\left|\beta_{1}\right|^{2}$, and it is straightforward to show that

$$
\beta_{1}=\left(\prod_{k=1}^{m}-\phi_{k}\right) \sum_{i=1}^{m} \frac{\left(\left|\phi_{i}\right|^{2}-1\right)}{\phi_{i}} .
$$

Suppose that $G_{d}(z)$ is obtained by discretizing a continuoustime plant $G(s)$ with a single real $\mathbb{C}^{+}$pole $p$ and a sampling period $T$ s. Then $G_{d}(z)$ has an unstable pole $\phi=e^{p T}$, and $\delta=4 e^{2 p T} \sinh ^{2}(p T)$. If $G(s)$ had a complex pair of $\mathbb{C}^{+}$poles, $x \pm j y$, then $\delta=16 e^{2 x T} \sinh ^{2}(x T) \cos ^{2}(y T)$.

Remark III.2: A recent paper by Elia gives a procedure for minimizing $\left\|K S_{d}\right\|_{H_{2}}$, where $S_{d}(z)=\left(1-z^{-1} F(z) K(z)\right)^{-1}$, $F(z)$ is the transfer function of a stable but possibly NMP communication channel, and $K(z)$ is a controller required to be open loop unstable [25, pp. 1481-1483]. In the special case $F(z)=1$, the procedure in [25] for minimizing this transfer

\footnotetext{
${ }^{5}$ This assumption may be relaxed, see the proof for details.
} 


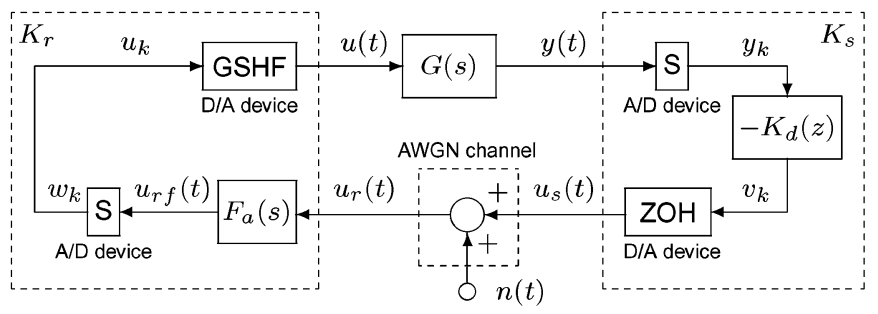

Fig. 6. Stabilization of an analog system via sampled-data output feedback over an analog AWGN channel

function provides an alternate approach to our Theorem III.2. Although stability robustness is not considered in [25], it is of interest to note that if the channel $F(z)$ has a NMP zero $\zeta$ and the controller $K(z)$ has a $\overline{\mathbb{D}}^{\mathrm{C}}$ pole $\phi$, then it follows from [15, Theorem 3.4.2] that the complementary sensitivity function of the resulting feedback system $T_{d}(z)=1-S_{d}(z)$ must satisfy

$$
\sup _{\theta}\left|T_{d}\left(e^{j \theta}\right)\right| \geq|\phi|\left|\frac{1-\zeta \phi}{\phi-\zeta}\right| \text {. }
$$

As in the continuous-time case, a large peak in $\left|T_{d}\left(e^{j \theta}\right)\right|$ will imply poor robustness and noise response (cf. the discussion of (21)). The factor of $|\phi|$ on the RHS of (36) is due to the single step time delay in the system, and will be large if the compensator pole lies well outside the unit circle. The second factor is due to the NMP zero, and will be large if the zero is close to the pole. In the context of [25], an unstable pole is used in $K$ to obtain a large data rate. However, (36) shows that a large unstable pole will cause robustness difficulties that will be worsened if any of the unstable poles also happens to lie near a NMP channel zero.

\section{LINEAR TIME-VARYING FEEDBACK STABILIZATION}

We saw in Section II that the presence of nonminimum phase zeros implies that the SNR required for stabilization with LTI output feedback is greater than that required for state feedback. In this section, we show that by using periodic linear time varying output feedback it is possible to stabilize an unstable NMP plant using a SNR arbitrarily close to that achievable with state feedback. In addition, we also show how (nonuniform in time) asymptotic stability, in the sense that $\lim _{k \rightarrow \infty} \mathrm{E}\left\{y_{k}^{2}\right\} \rightarrow 0$, can be achieved. These results demonstrate that, for a finite dimensional LTI plant, linear feedback suffices to achieve closed-loop stability with a SNR arbitrarily close to that consistent with the lowest data-rate required for stabilization [3], [7]. The price to be paid, as we shall discuss, is loss of stability robustness and higher sensitivity to disturbances.

\section{A. Stabilization Using Periodic LTV Feedback}

We shall use the sampled-data control scheme depicted in Fig. 6 (cf. Fig. 1), and whose components are defined as follows. Let the analog plant $G(s)$ have the minimal state variable realization (1) and $\mathbb{C}^{+}$poles $\left\{p_{i} ; i=1, \ldots, m\right\}$. The plant is discretized using a sampler with period $T$ and a generalized sampled data hold function (GSHF) [26] chosen so that $G_{d}(z)$, the transfer function of the discretized plant, has relative degree one and $(n-1)$ zeros lying in $\mathbb{D}$. The discrete control law is dynamic

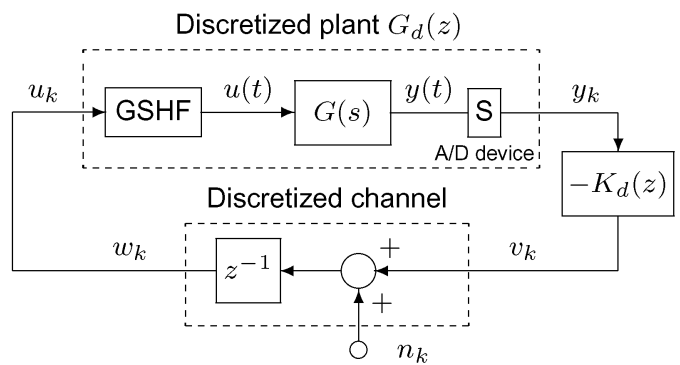

Fig. 7. Feedback system with discretized plant and channel.

output feedback applied to samples of the plant output: $V(z)=$ $-K_{d}(z) Y(z)$.

The analog AWGN communication channel is given by (2), where $n(t)$ is zero mean Gaussian white noise with power spectral density $\Phi$. The input to this channel is equal to the output sequence of the discrete controller passed through a zero-order hold $(\mathrm{ZOH})$

$$
u_{s}(t)=v_{k-1}, t \in[(k-1) T, k T) .
$$

The power in the channel input is, thus, equal to that in the controller output: $\left\|u_{s}\right\|_{\text {Pow }}=\mathrm{E}\left\{u_{s}^{2}(t)\right\}=\mathrm{E}\left\{v_{k}^{2}\right\}=\|v\|_{\text {Pow }}$. The output of the channel is passed through an averaging filter with transfer function $F_{a}(s)=\left(1-e^{-s T}\right) / s T$ and time response

$$
u_{r f}(t) \triangleq \frac{1}{T} \int_{t-T}^{t} u_{r}(\tau) d \tau
$$

and then sampled to obtain a discrete sequence $w_{k}=u_{r f}(k T)$. The AWGN channel in Fig. 6 may, thus, be reduced to a discrete channel with properties described as follows.

Lemma IV.1. (Discretized AWGN Channel): The discrete sequences $w_{k}$ and $v_{k}$ in Fig. 6 are related by the difference equation

$$
w_{k}=v_{k-1}+n_{k-1}
$$

where $n_{k}$ is a zero-mean Gaussian white noise process with variance $\sigma^{2}=\Phi / T$.

Proof: It follows from (2) and (38) that:

$$
w_{k}=\frac{1}{T} \int_{(k-1) T}^{k T} u_{s}(t) d t+\frac{1}{T} \int_{(k-1) T}^{k T} n(t) d t .
$$

Hence, (39) follows from (37) by defining the sequence $n_{k-1}$ from the second term on the RHS of (40). The statistical properties of the discrete noise sequence may be obtained by modifying the arguments of $[24, \S 10.6]$ or $[27, \S 9.4 .4]$.

The mapping from $v_{k}$ to $w_{k}$ in Fig. 6 is, thus, that of the discrete-time Gaussian channel in Fig. 7 with noise input $n_{k}$ and a one sample delay.

The transfer function between the noise $n_{k}$ and the channel input $v_{k}$ in Fig. 7 is equal to $-T_{d}(z)$, defined by

$$
T_{d}(z)=\frac{z^{-1} G_{d}(z) K_{d}(z)}{1+z^{-1} G_{d}(z) K_{d}(z)}
$$

and the power of the discrete channel input is equal to $\|v\|_{\text {Pow }}=$ $\left\|T_{d}\right\|_{H_{2}}^{2} \Phi / T$. Theorem III.2 may now be applied to minimize 
the power of the discretized channel input in Fig. 7 subject to the constraint of closed loop stability. As we have noted, the power of the discrete channel input is equal to that of the input to the AWGN channel in Fig. 6, and hence this procedure may be used to minimize the power of the analog AWGN channel input.

Theorem IV.2: Consider the sampled-data feedback system depicted in Fig. 7, denote the $\overline{\mathbb{D}}^{\mathrm{c}}$ poles of $G_{d}(z)$ by $\left\{\phi_{i}=\right.$ $\left.e^{p_{i} T} ; i=1, \ldots, m\right\}$, and define $T_{d}(z)$ by (41). Then

$$
\inf _{K_{d}(z) \in \mathcal{K}_{d}}\left\|T_{d}\right\|_{H_{2}}^{2}=\left(\prod_{i=1}^{m}\left|\phi_{i}\right|^{2}\right)-1+\delta
$$

where

$$
\delta=\prod_{i=1}^{m}\left|\phi_{i}\right|^{2}\left|\sum_{i=1}^{m} \frac{\left|\phi_{i}\right|^{2}-1}{\phi_{i}}\right|^{2}
$$

It follows that the feedback system can be stabilized, and the power constraint $\left\|u_{s}\right\|_{\text {Pow }}<\mathcal{P}$ satisfied, if and only if the SNR of the analog channel satisfies the lower bound

$$
\frac{\mathcal{P}}{\Phi}>\frac{\left(\prod_{i=1}^{m}\left|\phi_{i}\right|^{2}\right)-1+\delta}{T} .
$$

Proof: By construction, the plant $G_{d}(z)$ is minimum phase and has relative degree one. Applying Theorem III.2 to stabilize the series connection of $G_{d}(z)$ and the additional delay due to the discretized channel, thus, shows that the minimal value of $\left\|T_{d}\right\|_{H_{2}}^{2}$ is given by (33) with $\delta=\left|\beta_{1}\right|^{2}$ and $\beta_{1}$ given by (35). The SNR bound (44) follows by substituting (42) and using the fact that $\|v\|_{\text {Pow }}=\left\|u_{s}\right\|_{\text {Pow }}$.

Since $G_{d}(z)$ has relative degree one and no NMP zeros, the term $\delta$ in (44) represents the additional SNR required due to the one step delay in the discretized channel. Since a one step delay in the discrete domain corresponds to a $T$-second time delay in the continuous domain, it is plausible that this additional SNR should decrease with fast sampling.

Corollary IV.3: Assume that the SNR of the analog channel in Fig. 6 satisfies the bound

$$
\frac{\mathcal{P}}{\Phi}>\sum_{i=1}^{m} 2 \operatorname{Re}\left\{p_{i}\right\}
$$

Then there exists a sufficiently small $T$ and a stabilizing controller $K_{d}(z)$ such that input to the analog channel in Fig. 6 satisfies the power constraint $\left\|u_{s}\right\|_{\text {Pow }}<\mathcal{P}$.

Proof: The fact that $\phi_{i}=e^{p_{i} T}$ implies that ${ }^{6} \phi_{i}=1+$ $p_{i} T+O\left(T^{2}\right)$ for small values of $T$. It is, thus, straightforward to show that

$$
\begin{aligned}
\lim _{T \rightarrow 0} \frac{\left(\prod_{i=1}^{m}\left|\phi_{i}\right|^{2}\right)-1}{T} & =2 \sum_{i=1}^{m} \operatorname{Re}\left\{p_{i}\right\}, \text { and } \\
\lim _{T \rightarrow 0} \frac{\delta}{T} & =\lim _{T \rightarrow 0} \prod_{i=1}^{m}\left|\phi_{i}\right|^{2}\left|\sum_{i=1}^{m} \frac{\left|\phi_{i}\right|^{2}-1}{\phi_{i} \sqrt{T}}\right|^{2}=0 .
\end{aligned}
$$

\footnotetext{
${ }^{6}$ We use Landau's "big $O$ " notation for infinitesimal asymptotics as described in $[28$, p. 373$]$.
}

Corollary IV. 3 shows that the LTV feedback scheme in Fig. 6 allows the stabilization of an unstable NMP plant with an SNR arbitrarily close to that achievable with state feedback, which is consistent with the lowest data rate required under the only constraint of causality [7].

Let us suppose again that $G(s)$ has a single real $\mathbb{C}^{+}$pole $p$ and a single real NMP zero $z$. By appropriate choice of GSHF [26], it is possible to obtain a discretized plant with no NMP zeros, an unstable pole at $\phi=e^{p T}$, and relative degree $r=1$. The lower bound on admissible SNR (44), thus, becomes

$$
\frac{\mathcal{P}}{\Phi}>\frac{e^{2 p T}-1+4 e^{2 p T} \sinh ^{2}(p T)}{T} .
$$

For small values of $T, \sinh (p T)=p T+O\left(T^{3}\right)$, and, thus, the limit as $T \rightarrow 0$ of the RHS of (46) is equal to $2 p$, which is consistent with (45). For comparison, this limit should be compared with the SNR bound (21), which shows the potentially significant effects of the NMP zero.

\section{B. Robustness of the Proposed LTV Feedback Scheme}

We now use the framework developed in [29] and [30] to study robustness of the time-varying feedback system in Fig. 6 to uncertainty in the continuous-time plant. To do so, we use the fundamental complementary sensitivity function $T_{\text {fun }}(s)$ introduced in [29], and modified to the setting of Fig. 6. Denote the response function [31] of the GSHF by $H(s)$, note that the transfer function of the discretized channel in Fig. 7 is equal to $z^{-1}$, and define the discrete sensitivity function by $S_{d}(z)=\left(1+z^{-1} G_{d}(z) K_{d}(z)\right)^{-1}$. Then

$$
T_{\text {fun }}(s) \triangleq \frac{1}{T} G(s) H(s) S_{d}\left(e^{s T}\right) K_{d}\left(e^{s T}\right) e^{-s T} .
$$

As discussed in [29], [30], a large peak in $\left|T_{\text {fun }}(j \omega)\right|$ implies that stability robustness is poor. Theorem 4 of [29] implies that

$$
\sup _{\omega}\left|T_{\text {fun }}(j \omega)\right| \geq e^{\operatorname{Re}\{p\} T}\left|B_{z}^{-1}(p)\right|
$$

where $B_{z}(s)$ is defined by (20). The first term on the RHS of (47) is due to the one step delay in the discretized channel, and will converge to unity with fast sampling. The second term depends only on the relative location of $p$ to the NMP zeros of the plant, and will be very large if there exists an approximate pole/zero cancellation. Comparing (47) to (21), we see that the limitations imposed on the continuous-time response by the NMP zeros are the same with sampled-data control as they are with LTI control.

To summarize, with sampled-data control an unstable system can be stabilized with the same SNR as that available with state feedback, and, thus, the minimal channel capacity required for stabilization with time-varying control is identical, in units of bits/s, to the data rate derived by Nair and Evans [7]. However, in those cases for which time-varying control offers a significant reduction in SNR with respect to that for LTI control (i.e., those cases for which there exists a NMP zero close to an unstable pole), the robustness of the resulting design in the proposed LTV scheme will be poor by an amount that is also quantified by the proximity of the NMP zero to the unstable pole. 


\section{Asymptotic Stabilization Using LTV Feedback}

In the data rate limited setting, it has also been noted (see, for example, [5], [17]) that asymptotic stability can be attained, that is, $\mathrm{E}\left\{y_{k}^{2}\right\} \rightarrow 0$. The basic mechanism by which this is attained is the use of successively finer resolution in the encoding of the feedback information (with a consequent reduction in dynamic range). Here we show that this same mechanism can be used in our SNR constrained framework. For brevity, we state the results only in the continuous-time case, with discrete-time results following similarly.

Reconsider the general scheme depicted in Fig. 1, and let the plant $G(s)$ be stabilizable with output feedback within the SNR constraint (19). In view of (19) and Theorem II.2, take any controller $K_{1}(s)$ such that the corresponding complementary sensitivity function $T_{1}(s)=G(s) K_{1}(s) /\left(1+G(s) K_{1}(s)\right)$ satisfies the SNR constraint (14). Then for any $\alpha>0$ sufficiently small, we take a LTV control structure as follows:

$$
\begin{aligned}
u_{s}(t) & =e^{\alpha t} y(t) \\
u(t) & =-K_{1}(s) *\left(e^{-\alpha t} u_{r}(t)\right) \\
& =-K_{1}(s) *\left(y(t)+e^{-\alpha t} n(t)\right) .
\end{aligned}
$$

With regards to Fig. 1, this LTV control structure is given by $K_{s}$ as the LTV gain $K_{s}=e^{\alpha t}$, and $K_{r}$ as the transfer function $-K_{1}(s)$ preceded by the LTV gain $e^{-\alpha t}$.

If we let $h_{T_{1}}(t)$ be the impulse response of the transfer function $T_{1}(s)$ then we can evaluate sample paths of the output as

$$
y(t)=-\int_{0}^{t} h_{T_{1}}(t-\tau) n(\tau) e^{-\alpha \tau} d \tau
$$

and then, using standard properties of stochastic integrals (e.g., $[20, \S 5]$ ), we can show that $\mathrm{E}\left\{y^{2}(t)\right\}=$ $\Phi \int_{0}^{t} h_{T_{1}}^{2}(t-\tau) e^{-2 \alpha \tau} d \tau$. Thus, from (48), we have that

$$
\mathrm{E}\left\{u_{s}^{2}(t)\right\}=\Phi \int_{0}^{t} h_{T_{1}}^{2}(t-\tau) e^{2 \alpha(t-\tau)} d \tau
$$

and, therefore, $\lim _{t \rightarrow \infty} \mathrm{E}\left\{u_{s}^{2}(t)\right\}=\Phi\left\|T_{1}(s-\alpha)\right\|_{H_{2}}^{2}$. Now, provided that $\alpha$ is smaller than the absolute stability margin of $T_{1}$, that is, that $T_{1}(s-\alpha)$ is analytic in $\overline{\mathbb{C}}^{+}$, the expression (50) is well defined, and also, as we take $\alpha \rightarrow 0^{+}$we have that $\left\|T_{1}(s-\alpha)\right\|_{H_{2}}^{2} \rightarrow\left\|T_{1}(s)\right\|_{H_{2}}^{2}$. It, therefore, follows that for sufficiently small $\alpha$ we satisfy the SNR constraint (14), and in addition

$$
\mathrm{E}\left\{y^{2}(t)\right\} \rightarrow e^{-2 \alpha t} \Phi\left\|T_{1}(s-\alpha)\right\|_{H_{2}}^{2} \rightarrow 0
$$

Note, however, that this strategy is infinitely sensitive to additive noise. For example, suppose we add an arbitrarily small amount of input (or process) noise $n_{u}$ to the input of the plant: $y(t)=G(s) *\left(u(t)+n_{u}(t)\right)$. In this case, (49) becomes $y(t)=-\int_{0}^{t} h_{T_{1}}(t-\tau) n(\tau) e^{-\alpha \tau} d \tau+\int_{0}^{t} h_{G S_{1}}(t-\tau) n_{u}(\tau) d \tau$, where $h_{G S_{1}}$ denotes the impulse response of the closed loop transfer function $G(s) S_{1}(s)$ relating the input noise to the plant output. Then assuming $n_{u}$ is white with power spectral density
$\Phi_{n_{u}}$, and is uncorrelated with the channel noise $n(t),(51)$ becomes

$$
\mathrm{E}\left\{y^{2}(t)\right\} \rightarrow e^{-2 \alpha t} \Phi\left\|T_{1}(s-\alpha)\right\|_{H_{2}}^{2}+\Phi_{n_{u}}\left\|G(s) S_{1}(s)\right\|_{H_{2}}^{2} .
$$

From (52) and (48), for any $\Phi_{n_{u}}>0$, no matter how small, the transmitted power in this case diverges: $\mathrm{E}\left\{u_{s}^{2}(t)\right\} \rightarrow e^{2 \alpha t} \mathrm{E}\left\{y^{2}(t)\right\} \rightarrow+\infty$.

\section{CONCLUSION}

In this paper, we have considered a framework for discussing control over a communication channel based on SNR constraints. We have focused particularly on the feedback stabilization of an open loop unstable plant via a channel with a SNR constraint. By examining the simple case of a linear time invariant plant and an AWGN channel, we have derived necessary and sufficient conditions on the SNR for feedback stabilization with an LTI controller. These conditions have been expounded in continuous and discrete-time, for both state feedback and output feedback cases. Interestingly, for both the minimum phase and the state feedback cases, our SNR results, together with the associated Shannon capacity formula, parallel directly previous results on data rate limited stabilization.

Beyond previous results on data rate limits, we have shown that stabilization of an unstable nonminimum phase LTI plant via an LTI controller does result in additional SNR demands, as compared to the minimum phase case. This additional demand on SNR for LTI stabilization may be made vanishingly small by the use of fast sampling and LTV control schemes such as those based on GSHFs. However, in such cases, the use of LTV control to reduce the required SNR will necessarily result in poor robustness and sensitivity properties.

As compared to other approaches, simplicity is arguably a highlight of the proposed SNR constraint framework, which inherently captures channel noise effects, while dispensing with the use of quantizers and time-varying coding/decoding schemes. Notwithstanding this simplicity, we believe this framework offers considerable scope for a variety of control over communication links problems. Future extensions to this work include more general plants, such as continuous-time plants with time delays (see [16] for preliminary work in this direction) and multivariable systems, channel models with bandwidth limitations, and control problems beyond that of stabilization.

\section{APPENDIX}

\section{A. Proof of Theorem II.1}

We translate the problem of minimizing (7) into a minimum energy stabilization problem: find a state feedback $u=-K x$ to minimize the cost function $J(x(0))=\int_{0}^{\infty} u^{2}(t) d t$ subject to the constraints that the system $\dot{x}=A x+B u$ be asymptotically stable and that $x(0)=B$. The control signal for this initial condition is given by $u(t)=$ $-K e^{(A-B K) t} B$, and, thus, Parseval's theorem implies that $J(B)=(1 / 2 \pi) \int_{-\infty}^{\infty}\left|K(j \omega-A+B K)^{-1} B\right|^{2} d \omega$. It is easy to show that $T^{s f}$ defined by (5) satisfies the identity $T^{s f}(s)=K(s I-A+B K)^{-1} B$, and, thus, $J(B)=\left\|T^{s f}\right\|_{H_{2}}^{2}$. 
Assume temporarily that $A$ has no eigenvalues on the $j \omega$ axis. Then the system may be assumed to have the modal form $\dot{x}=\left[\begin{array}{cc}A_{s} & 0 \\ 0 & A_{u}\end{array}\right] x+\left[\begin{array}{c}B_{s} \\ B_{u}\end{array}\right] u$, where $A_{s}$ and $-A_{u}$ are both Hurwitz. The assumption that $(A, B, C)$ is minimal implies that $\left(A_{u}, B_{u}\right)$ is controllable and, thus, the minimum energy stabilization problem has the well-defined solution $K=B^{\prime} P$, where $P$ is the unique positive semidefinite solution to the algebraic Riccati equation $0=A^{\prime} P+P A-P B B^{\prime} P$. It follows from [32, Lemma 2] that $P$ has the form $P=\operatorname{diag}\left\{0, P_{u}\right\}$, where $P_{u}$ is the unique positive definite solution to the reduced Riccati equation

$$
0=A_{u}^{\prime} P_{u}+P_{u} A_{u}-P_{u} B_{u} B_{u}^{\prime} P_{u}
$$

The optimal cost for $x(0)=B$ is, thus

$$
\begin{aligned}
J^{*}(B) & =B^{\prime} P B=B_{u}^{\prime} P_{u} B_{u}=\operatorname{trace} P_{u}^{1 / 2} B_{u} B_{u}^{\prime} P_{u}^{1 / 2} \\
& =\operatorname{trace}\left(P_{u}^{-1 / 2} A_{u}^{\prime} P_{u}^{1 / 2}+P_{u}^{1 / 2} A_{u} P_{u}^{-1 / 2}\right) \\
& =2 \operatorname{trace} A_{u}=2 \sum_{i=1}^{m} \operatorname{Re}\left\{p_{i}\right\}
\end{aligned}
$$

which, since $J(B)=\left\|T^{s f}\right\|_{H_{2}}^{2}$, proves (7). It follows from (53) that $-P_{u}^{-1} A_{u}^{\prime} P_{u}=A_{u}-B_{u} B_{u}^{\prime} P_{u}$, and, thus, that the closed loop eigenvalues are the union of the eigenvalues of $A_{s}$ and $-A_{u}$.

If $A$ has $j \omega$-axis eigenvalues, let $A(\epsilon)=A+\epsilon I$ with $\varepsilon>0$ such that $A(\varepsilon)$ has the same number of $\mathbb{C}^{-}$eigenvalues as $A$, but no eigenvalues on the $j \omega$-axis. Applying the above procedure and letting $\varepsilon \rightarrow 0$ shows that $j \omega$-axis eigenvalues do not contribute to the optimal cost.

Although $j \omega$ axis eigenvalues do not contribute to the optimal cost, they do imply that a stabilizing state feedback achieving the infimum (7) does not exist. Such a feedback may be approximated arbitrarily closely by finding $K_{\alpha}$ to minimize $J_{\alpha}=$ $\int_{0}^{\infty}\left(\alpha^{2} x^{\prime} x+u^{2}\right) d t$ for $\alpha$ sufficiently small. As is well known (cf. [33]), in the limit as $\alpha \rightarrow 0$, the eigenvalues of $A-B K_{\alpha}$ approach the $\mathbb{C}^{-}$mirror images of the $\mathbb{C}^{+}$eigenvalues of $A$, and the $\overline{\mathbb{C}}^{-}$eigenvalues of $A$. If $A$ has no eigenvalues on the $j \omega$ axis, then this result agrees with that above. If $A$ does have such eigenvalues, then those eigenvalues of $A-B K_{\alpha}$ that converge to the $j \omega$ axis are guaranteed to be stable, thus, yielding a stabilizing control law $u_{\alpha}(t)$ whose cost $\int_{0}^{\infty} u^{2}(t) d t$ approximates that of a control law that moves only the $\mathbb{C}^{+}$eigenvalues of $A$ and leaves the rest fixed.

\section{B. Proof of Theorem II.2}

We apply a technique used in [34]. Define the spaces $L_{2}$, $H_{2}, H_{2}^{\perp}$, and $H_{\infty}$ as in [35]. Consider a coprime factorization $G=N / M$, where $N, M \in H_{\infty}$. The Youla parametrization of all controllers that stabilize $G$ is given by $K=(X+M Q) /(Y-$ $N Q$ ), where $Q, X, Y \in H_{\infty}$ and $X$ and $Y$ satisfy the Bezout identity $N X+M Y=1$ [35]. Substituting these factorizations for $G$ and $K$ into (13) and applying the Bezout identity shows that $T=1-M(Y-N Q)$. Hence the problem of finding $K$ to minimize (16) reduces to that of finding $Q$ to minimize $\| 1-$ $M Y+M N Q \|_{H_{2}}^{2}$. Further factorize $M=B_{p} M_{0}$, where the
Blaschke product $B_{p}$ is given by (15), and satisfies $\left|B_{p}(j \omega)\right|=$ $1, \forall \omega$ and $B_{p}^{-1} \in H_{2}^{\perp}$. Hence

$$
\begin{aligned}
& \inf _{Q \in H_{\infty}}\|1-M Y+M N Q\|_{H_{2}}^{2} \\
& \quad=\inf _{Q \in H_{\infty}}\left\|\left(B_{p}^{-1}-1\right)+\left(1-M_{0} Y+M_{0} N Q\right)\right\|_{L_{2}}^{2} \\
& =\left\|B_{p}^{-1}-1\right\|_{L_{2}}^{2}+\inf _{Q \in H_{\infty}}\left\|1-M_{0} Y+M_{0} N Q\right\|_{H_{2}}^{2}
\end{aligned}
$$

The first term on the RHS of (54) may be evaluated using the residue theory [15], [28], [36]. Let $C_{R}$ denote a contour consisting of the imaginary axis $\{s=j \omega,-R \leq \omega \leq R\}$ together with a large semicircle in $\mathbb{C}^{+}\left\{s=R e^{j \theta},-\pi / 2 \leq \theta \leq\right.$ $\pi / 2\}$, and traversed in the clockwise direction. Then

$$
\begin{gathered}
\left\|B_{p}^{-1}-1\right\|_{L_{2}}^{2}=\frac{1}{2 \pi} \int_{-\infty}^{\infty}\left(B_{p}^{-1}(j \omega)-1\right)\left(B_{p}^{-1}(-j \omega)-1\right) d \omega \\
=\lim _{R \rightarrow \infty} \frac{1}{2 \pi j} \oint_{C_{R}}\left(B_{p}^{-1}(s)-1\right) \\
\times\left(B_{p}^{-1}(-s)-1\right) d s
\end{gathered}
$$

because the integrand has two more poles than zeros, and, thus, the limiting value of the integral over the semicircle will equal zero [15, Example A.4.1]. Let $R$ be sufficiently large so that $C_{R}$ encloses all the $\mathbb{C}^{+}$poles of the integrand of (55). By (15), this integrand reduces to $f(s) \triangleq 2-B_{p}(s)-B_{p}^{-1}(s)$, and we see that the only $\mathbb{C}^{+}$poles of $f(s)$ are those of $B_{p}^{-1}$, which are located at $\left\{p_{i} ; i=1, \ldots, m\right\}$. Hence the contour integral of the term $2-B_{p}(s)$ is equal to zero, and we need only to evaluate the residues of $B_{p}^{-1}$, which we shall denote by $\operatorname{Res}\left\{B_{p}^{-1}, p_{i}\right\}$. The residue theorem [15, Theorem A.9.1] applied to the contour $C_{R}$ yields from (55)

$$
\left\|B_{p}^{-1}-1\right\|_{L_{2}}^{2}=-\sum_{i=1}^{m} \operatorname{Res}\left\{B_{p}^{-1} p_{i}\right\} .
$$

An identity from [36, p. 122] shows that $\left\|B_{p}^{-1}-1\right\|_{L_{2}}^{2}=$ $\operatorname{Res}\left\{B_{p}^{-1}, \infty\right\}$, where $\operatorname{Res}\left\{B_{p}^{-1}, \infty\right\}$ is equal to the coefficient of $\zeta$ in the power series expansion of $B_{p}^{-1}(1 / \zeta)$ about $\zeta=0$ [28, p. 233]. Setting $s=1 / \zeta$ in (15) and expanding each term in the product in powers of $\zeta$ yields $B_{p}^{-1}(1 / \zeta)=\prod_{i=1}^{m}\left(1+2 \operatorname{Re}\left\{p_{i}\right\} \zeta+\zeta^{2}\left|p_{i}\right|^{2}+O\left(\zeta^{3}\right)\right)$, and, thus, $\left\|B_{p}^{-1}-1\right\|_{L_{2}}^{2}=2 \sum_{i=1}^{m} \operatorname{Re}\left\{p_{i}\right\}$.

It remains to evaluate the second term on the RHS of (54). Factor $N(s)=B_{z}(s) N_{m}(s)$, where $B_{z}(s)$ is the Blaschke product (20) and $N_{m}$ is the minimum phase factor of $N$. Decompose $B_{z}^{-1}\left(1-M_{0} Y\right)=\Gamma+\Gamma^{\perp}$, where $\Gamma \in H_{2}$ and $\Gamma^{\perp} \in H_{2}^{\perp}$. Because Blaschke products are all-pass

$$
\begin{aligned}
& \inf _{Q \in H_{\infty}}\left\|1-M_{0} Y+M_{0} N Q\right\|_{H_{2}}^{2} \\
& \quad=\inf _{Q \in H_{\infty}}\left\|B_{z}^{-1}\left(1-M_{0} Y\right)+\left(M_{0} N_{m} Q\right)\right\|_{L_{2}}^{2} \\
& \quad=\left\|\Gamma^{\perp}\right\|_{H_{2}^{\perp}}^{2}+\inf _{Q \in H_{\infty}}\left\|\Gamma+M_{0} N_{m} Q\right\|_{H_{2}}^{2} .
\end{aligned}
$$


By construction, $M_{0}$ and $N_{m}$ are minimum phase. Hence the second term on the RHS of (56) can be set to zero using $Q=$ $-\Gamma / M_{0} N_{m}$, where $Q$ has no $\mathbb{C}^{+}$poles but may be improper and will have poles on the $j \omega$ axis if $G$ has either poles or zeros there. In that case, a construction from [37, Lemma 10, p. 171] may be used to find an approximation $Q_{\epsilon} \in H_{\infty}$ such that $\left\|\Gamma+M_{0} N_{m} Q_{\epsilon}\right\|_{H_{2}}^{2}<\epsilon$ for an arbitrary $\epsilon>0$.

The final step in the proof is to compute $\left\|\Gamma^{\perp}\right\|_{H_{2}^{\perp}}^{2}$ in (56). To do so, note that the $\mathbb{C}^{+}$poles of $\Gamma^{\perp}$ are precisely the NMP zeros of $G$. Since these are assumed distinct, we consider the partial fraction expansion ${ }^{7} \Gamma^{\perp}(s)=\sum_{\ell=1}^{q}\left(\gamma_{\ell} /\left(s-z_{\ell}\right)\right)$, where $\gamma_{\ell} \triangleq \operatorname{Res}\left\{B_{z}^{-1}, z_{\ell}\right\}\left(1-M_{0}\left(z_{\ell}\right) Y\left(z_{\ell}\right)\right)$. It is straightforward to show that $\operatorname{Res}\left\{B_{z}^{-1}, z_{\ell}\right\}=2 \operatorname{Re}\left\{z_{\ell}\right\} \prod_{\substack{k=1 \\ k \neq \ell}}^{q}\left(z_{\ell}+\bar{z}_{k}\right) /\left(z_{\ell}-z_{k}\right)$, and it follows from the Bezout identity that $M_{0}\left(z_{\ell}\right) Y\left(z_{\ell}\right)=$ $B_{p}^{-1}\left(z_{\ell}\right)$. Hence, $\gamma_{\ell}$ satisfies (18). The residue theorem applied to a clockwise contour implies that

$$
\left\|\Gamma^{\perp}\right\|_{H_{2}^{\perp}}^{2}=-\sum_{\ell=1}^{q} \operatorname{Res}\left\{\Gamma^{\perp}(s) \bar{\Gamma}^{\perp}(-s) z_{\ell}\right\}
$$

where $\operatorname{Res}\left\{\Gamma^{\perp}(s) \bar{\Gamma}^{\perp}(-s), z_{\ell}\right\}=\gamma_{\ell} \sum_{k=1}^{q}-\bar{\gamma}_{k} /\left(z_{\ell}+\bar{z}_{k}\right)$. Summing the last expression over $\ell$ and substituting into (57) shows that $\left\|\Gamma^{\perp}\right\|_{H_{2}^{\perp}}^{2}=\eta$, defined in (17).

\section{Proof of Theorem III.1}

Following the idea of the proof in Appendix A, we translate (26) into the problem: find a state feedback $v_{k}=-K_{d} x_{k}$ to minimize the cost $J\left(x_{0}\right)=\sum_{k=0}^{\infty} u_{k}^{2}$ and such that the system $x_{k+1}=A_{d} x_{k}+B_{d} v_{k}$ be asymptotically stable, with $x_{0}=B_{d}$. The corresponding control signal is $u_{k}=-K_{d}\left(A_{d}-B_{d} K_{d}\right)^{k} B_{d}$, which by Parseval yields $J\left(B_{d}\right)=(1 / 2 \pi) \int_{-\pi}^{\pi}\left|K_{d}\left(e^{j \theta} I-A_{d}+B_{d} K_{d}\right)^{-1} B_{d}\right|^{2} d \theta$. Then, from (24), $T_{d}^{s f}(z)=K_{d}\left(z I-A_{d}+B_{d} K_{d}\right)^{-1} B_{d}$, and, thus, $J\left(B_{d}\right)=\left\|T_{d}^{s f}\right\|_{H_{2}}^{2}$.

Assuming temporarily that $A_{d}$ has no eigenvalues on the unit circle, let $A_{d}$ and $B_{d}$ be given in the modal form $A_{d}=\left[\begin{array}{cc}A_{s} & 0 \\ 0 & A_{u}\end{array}\right], B=\left[\begin{array}{l}B_{s} \\ B_{u}\end{array}\right]$, where $A_{s}$ and $A_{u}^{-1}$ are Schur. Because $\left(A_{d}, B_{d}, C_{d}\right)$ is minimal, the pair $\left(A_{u}, B_{u}\right)$ is controllable and the minimum energy stabilization problem has a well defined solution $K=\left(1+B^{\prime} S B\right)^{-1}$ where $S$ is the unique symmetric and positive semidefinite solution of the discrete algebraic Riccati equation $0=A_{d}^{\prime} S A_{d}-S-A_{d}^{\prime} S B_{d}\left(1+B_{d}^{\prime} S B_{d}\right)^{-1} B_{d}^{\prime} S A_{d}$. It can be shown that $S$ has the form $S=\operatorname{diag}\left\{0, S_{u}\right\}$, where $S_{u}$ is the unique symmetric and positive definite solution of the reduced order discrete algebraic Riccati equation

$$
0=A_{u}^{\prime} S_{u} A_{u}-S_{u}-A_{u}^{\prime} S_{u} B_{u}\left(1+B_{u}^{\prime} S_{u} B_{u}\right)^{-1} B_{u}^{\prime} S_{u} A_{u} .
$$

\footnotetext{
${ }^{7}$ This is the only place where the assumption of distinct zeros is used. If it does not apply, then the partial fraction expansion may be modified accordingly.
}

Then the minimum energy state feedback gain is given as $K_{d}=$ $\left[0 \quad\left(1+B_{u}^{\prime} S_{u} B_{u}\right)^{-1} B_{u}^{\prime} S_{u} A_{u}\right]$ and yields the closed loop spectrum

$$
\begin{aligned}
\sigma & \left\{A_{d}-B_{d} K_{d}\right\} \\
& =\sigma\left\{\left[\begin{array}{cc}
A_{s} & 0 \\
0 & A_{u}
\end{array}\right]-\left[\begin{array}{cc}
0 & B_{s}\left(1+B_{u}^{\prime} S_{u} B_{u}\right)^{-1} B_{u}^{\prime} S_{u} A_{u} \\
0 & B_{u}\left(1+B_{u}^{\prime} S_{u} B_{u}\right)^{-1} B_{u}^{\prime} S_{u} A_{u}
\end{array}\right]\right\} \\
& =\sigma\left\{A_{s}\right\} \cup \sigma\left\{A_{u}-B_{u}\left(1+B_{u}^{\prime} S_{u} B_{u}\right)^{-1} B_{u}^{\prime} S_{u} A_{u}\right\} \\
& =\sigma\left\{A_{s}\right\} \cup \sigma\left\{S_{u}^{-1} A_{u}^{\prime-1} S_{u}\right\}=\sigma\left\{A_{s}\right\} \cup \sigma\left\{A_{u}^{-1}\right\}
\end{aligned}
$$

which follows from (58).

On the other hand, by the Matrix Inversion Lemma we have that $A_{d}-B_{d} K_{d}=A_{d}-B_{d}\left(1+B_{d}^{\prime} S B_{d}\right)^{-1} B_{d}^{\prime} S A_{d}=$ $\left(I+B_{d} B_{d}^{\prime} S\right)^{-1} A_{d}$, which together with (59) implies that $\operatorname{det}\left\{A_{s}\right\} \operatorname{det}\left\{A_{u}^{-1}\right\}=\operatorname{det}\left\{\left(I+B_{d} B_{d}^{\prime} S\right)^{-1} A_{d}\right\}=$ $\left(1+B_{d}^{\prime} S B_{d}\right)^{-1} \operatorname{det}\left\{A_{s}\right\} \operatorname{det}\left\{A_{u}\right\}$ Hence, $\left(1+B_{d}^{\prime} S B_{d}\right)=$ $\operatorname{det}\left\{A_{u}\right\}^{2} \Leftrightarrow J\left(B_{d}\right)=B_{d}^{\prime} S B_{d}=\left(\prod_{i=1}^{m}\left|\phi_{i}\right|^{2}\right)-1$, completing the proof.

If $A_{d}$ has eigenvalues on the unit circle, it can be argued (as in Appendix A) that although the minimum energy solution does not stabilize the eigenvalues on the unit circle, they do not contribute to the cost and can be stabilized by an approximate solution that incurs a cost arbitrarily close to that of the minimum energy solution.

\section{Proof of Theorem III.2}

We proceed as in the proof of the continuous-time result in Appendix $\mathrm{B}$, and consider the function spaces $L_{2}(\mathbb{D}), H_{2}(\mathbb{D}), H_{2}^{\perp}(\mathbb{D})$, and $H_{\infty}(\mathbb{D})$, whose stability region is the open unit disk. Introduce a coprime factorization $G_{d}=N / M$, and the parametrization of all stabilizing controllers $K_{d}=(X+M Q) /(Y-N Q)$, where $X$ and $Y$ satisfy the Bezout identity $N X+M Y=1$. It follows that $T_{d}=1-M(Y-N Q)$. Further factorize $M=B_{\phi} M_{0}$, where $B_{\phi}$ is the Blaschke product (31). It follows from the Bezout identity that $B_{\phi}^{-1}$ and $M_{0} Y$ have power series expansions at infinity of the form

$$
B_{\phi}^{-1}(z)=\sum_{k=0}^{\infty} \beta_{k} z^{-k}
$$

and

$$
M_{0}(z) Y(z)=\sum_{k=0}^{r-1} \beta_{k} z^{-k}+\sum_{k=r}^{\infty} \alpha_{k} z^{-k}
$$

where $\beta_{k}$ is defined by (32). Since $B_{\phi}$ is all-pass of unit magnitude, $B_{\phi}^{-1} \in H_{2}^{\perp}, N$ has relative degree $r$, and the set $\left\{z^{-k} ; k=\right.$ $0, \ldots, \infty\}$ forms an orthonormal basis for $H_{2}$, it follows that

$$
\begin{aligned}
\left\|T_{d}\right\|_{H_{2}}^{2}= & \left\|B_{\phi}^{-1}-M_{0} Y+M_{0} N Q\right\|_{L_{2}}^{2} \\
= & \left\|B_{\phi}^{-1}(z)-\sum_{k=0}^{r-1} \beta_{k} z^{-k}\right\|_{L_{2}} \\
& +\left\|-\sum_{k=r}^{\infty} \alpha_{k} z^{-k}+M_{0}(z) N(z) Q(z)\right\|_{H_{2}}^{2} .
\end{aligned}
$$


The first term on the RHS of (61) may be further decomposed as

$$
\begin{array}{r}
\left\|B_{\phi}^{-1}(z)-\sum_{k=0}^{r-1} \beta_{k} z^{-k}\right\|_{L_{2}}=\left\|B_{\phi}^{-1}(z)-\beta_{0}\right\|_{L_{2}}^{2} \\
+\left\|\sum_{k=1}^{r-1} \beta_{k} z^{-k}\right\|_{L_{2}}^{2} .
\end{array}
$$

Orthonormality of the functions $\left\{z^{-k}\right\}$ implies that the second term on the RHS of (62) is equal to $\sum_{k=1}^{r-1}\left|\beta_{k}\right|^{2}$. The first term on the RHS of (62) may be evaluated by using the residue theorem applied to a contour $C$ consisting of the unit circle traversed counterclockwise (cf. similar manipulations in Appendix B)

$$
\begin{aligned}
\left\|B_{\phi}^{-1}(z)-\beta_{0}\right\|_{L_{2}}^{2}= & \frac{1}{2 \pi} \int_{-\pi}^{\pi}\left(B_{\phi}^{-1}\left(e^{j \theta}\right)-\beta_{0}\right) \\
& \times\left(B_{\phi}^{-1}\left(e^{-j \theta}\right)-\beta_{0}\right) d \theta \\
= & \frac{1}{2 \pi j} \oint_{C}\left(B_{\phi}^{-1}(z)-\beta_{0}\right) \\
& \times\left(B_{\phi}^{-1}\left(z^{-1}\right)-\beta_{0}\right) z^{-1} d z \\
= & \frac{1}{2 \pi j} \oint_{C}\left(\beta_{0}^{2}-\beta_{0} B_{\phi}(z)\right) z^{-1} d z \\
= & \beta_{0} \operatorname{Res}\left\{\left(\beta_{0}-B_{\phi}(z)\right) z^{-1}, \infty\right\} .
\end{aligned}
$$

It is straightforward to verify that $\beta_{0}=(-1)^{m} \prod_{i=1}^{m} \phi_{i}$. The coefficient of $\zeta$ in the expansion of $\zeta B_{\phi}\left(\zeta^{-1}\right)$ near $\zeta=0$ is equal to $1 / \beta_{0}$, and, thus, since complex poles appear in conjugate pairs, $\left\|B_{\phi}^{-1}(z)-\beta_{0}\right\|_{L_{2}}^{2}=\prod_{i=1}^{m}\left|\phi_{i}\right|^{2}-1$. This proves that $\left\|T_{d}\right\|_{H_{2}}$ is equal to the sum of two terms that do not depend on the controller, $\sum_{k=1}^{r-1}\left|\beta_{k}\right|^{2}+\prod_{i=1}^{m}\left|\phi_{i}\right|^{2}-1$, plus the second term on the RHS of (61), which may be minimized by choice of $Q$.

Denote the Blaschke product of nonminimum phase plant zeros by $B_{\zeta}(z)=\prod_{k=1}^{q}\left(z-\zeta_{k}\right) /\left(1-z \bar{\zeta}_{k}\right)$, factorize $N(z)=$ $N_{m}(z) B_{\zeta}(z)$, and define

$$
B_{\zeta}^{-1}(z) \sum_{k=r}^{\infty} \alpha_{k} z^{-k}=\Gamma(z)+\Gamma^{\perp}(z)
$$

where $\Gamma(z) \in H_{2}(\mathbb{D})$ and $\Gamma^{\perp}(z) \in H_{2}^{\perp}(\mathbb{D})$. The second term on the RHS of (61) reduces to

$$
\begin{aligned}
& \left\|\sum_{k=r}^{\infty} \alpha_{k} z^{-k}+M_{0}(z) N(z) Q(z)\right\|_{L_{2}}^{2} \\
& \quad=\left\|B_{\zeta}^{-1}(z) \sum_{k=r}^{\infty} \alpha_{k} z^{-k}+M_{0}(z) N_{m}(z) Q(z)\right\|_{L_{2}}^{2} \\
& \quad=\left\|\Gamma^{\perp}\right\|_{H_{2}^{\perp}}^{2}+\left\|\Gamma+M_{0} N_{m} Q\right\|_{H_{2}}^{2} .
\end{aligned}
$$

Since $N_{m}$ has relative degree $r$ and $M_{0}$ has relative degree zero, it follows that if $G_{d}$ has no poles or zeros on the $j \omega$ axis, then the second term on the RHS of (64) may be set equal to zero by defining $Q=-\Gamma / M_{0} N_{m} \in H_{\infty}(\mathbb{D})$. If such zeros are present, then the technique of [37] used in Appendix B may be modified to construct an approximation to $Q$.

The final step in the proof is to compute $\left\|\Gamma^{\perp}\right\|_{H_{2}}^{2}$ in (64). The $\overline{\mathbb{D}}^{c}$ poles of $\Gamma^{\perp}$ are precisely the NMP zeros of $G_{d}$. Since these are assumed distinct, introduce the partial fraction expansion 8 $\Gamma^{\perp}(z)=\sum_{\ell=1}^{q} \gamma_{\ell} /\left(z-\zeta_{\ell}\right)$, where $\gamma_{\ell}$ is the residue of $\Gamma^{\perp}$ at $z_{\ell}$. It follows from (60), (63), and the Bezout identity that

$$
\gamma_{\ell}=\operatorname{Res}\left\{B_{\zeta}^{-1} \zeta_{\ell}\right\}\left(B_{\phi}^{-1}\left(\zeta_{\ell}\right)-\sum_{k=0}^{r-1} \beta_{k} \zeta_{\ell}^{-k}\right) .
$$

It is easy to show that $\operatorname{Res}\left(B_{\zeta}^{-1} \zeta_{\ell}\right)=\left(1-\left|\zeta_{\ell}\right|^{2}\right) \prod_{\substack{k=1 \\ k \neq \ell}}^{q}(1-$ $\left.\zeta_{\ell} \bar{\zeta}_{k}\right) /\left(\zeta_{\ell}-\zeta_{k}\right)$. The residue theorem applied to the unit circle traversed clockwise implies that

$$
\left\|\Gamma^{\perp}\right\|_{H_{2}^{\perp}}^{2}=-\sum_{\ell=1}^{q} \operatorname{Res}\left\{z^{-1} \Gamma^{\perp}(z) \bar{\Gamma}^{\perp}\left(z^{-1}\right) \zeta_{\ell}\right\}
$$

where $\operatorname{Res}\left\{z^{-1} \Gamma^{\perp}(z) \bar{\Gamma}^{\perp}\left(z^{-1}\right), \zeta_{\ell}\right\}=\sum_{i=1}^{q} \gamma_{\ell} \bar{\gamma}_{i} /\left(1-\zeta_{\ell} \bar{\zeta}_{i}\right)$. The last expression together with (65) and (66) imply that $\eta$ in (34) has the stated form.

\section{REFERENCES}

[1] P. Antsaklis and J. Baillieul, Eds., IEEE Trans. Autom. Control. Special Issue on Networked Control Syst., vol. 49, no. 9, Sep. 2004.

[2] J. Baillieul, "Feedback coding for information based control: Operating near the data rate limit," in Proc. 41 st IEEE Conf. Decision Contr., Las Vegas, NV, 2002.

[3] G. N. Nair and R. E. Evans, "Stabilizability of stochastic linear systems with finite feedback data rates," SIAM J. Contr. Optimiz., vol. 43, no. 2, pp. 413-436, Jul. 2004.

[4] S. Tatikonda and S. M. Mitter, "Control under communication constraints," IEEE Trans. Autom. Control, vol. 49, no. 7, pp. 1056-1068, Jul. 2004.

[5] A. S. Matveev and A. V. Savkin, "An analogue for shannon information theory for networked control systems. Stabilization via a noisy discrete channel," in Proc. 43rd IEEE Conf. Decision Control, The Bahamas, 2004.

[6] S. Yüksel and T. Basar, "Coding and control over discrete noisy forward and feedback channels," in Proc. 44th IEEE Conf. Decision and Control and the Europ. Control Conf. 2005, Seville, Spain, Dec. 2005, pp. 2517-2522.

[7] G. N. Nair and R. J. Evans, "Exponential stabilisability of finite-dimensional linear systems with limited data rates," Automatica, vol. 39, no. 4, pp. 585-593, Apr. 2003.

[8] C. E. Shannon, "A mathematical theory of communication," Bell Sys. Tech. J., vol. 27, pp. 379-423, 1948, 623-656.

[9] C. E. Shannon, "Communication in the presence of noise," Proc. IRE, vol. 37, pp. 10-21, 1949.

[10] R. G. Gallager, Information Theory and Reliable Communication. New York: Wiley, 1968.

[11] T. M. Cover and J. A. Thomas, Elements of Information Theory. New York: Wiley, 1991.

[12] J. S. Freudenberg and D. P. Looze, "Right half plane poles and zeros and design tradeoffs in feedback systems," IEEE Trans. Autom. Control, vol. 30, no. 6, pp. 555-565, Jun. 1985.

[13] D. P. Looze and J. S. Freudeberg, "Limitations of feedback properties imposed by open-loop right half plane poles," IEEE Trans. Autom. Control, vol. 36, no. 6, pp. 736-739, Jun. 1991.

[14] R. H. Middleton, "Trade-offs in linear control systems design," Automatica, vol. 27, no. 2, pp. 281-292, Mar. 1991.

[15] M. M. Seron, J. H. Braslavsky, and G. C. Goodwin, Fundamental Limitations in Filtering and Control. New York: Springer, 1997.

[16] J. H. Braslavsky, R. H. Middleton, and J. Freudenberg, "Effects of time delay on feedback stabilisation over signal-to-noise ratio constrained channels," in Proc. 16th IFAC World Congress, Prague, Czech Republic, Jul. 2005.

${ }^{8}$ This is the only place where the assumption of distinct zeros is used. If it does not apply, then the partial fraction expansion may be modified accordingly. 
[17] G. N. Nair and R. E. Evans, "Stabilization with data-rate limited feedback: Tightest attainable bounds," Syst. Contr. Lett., vol. 41, pp. 49-56, 2000.

[18] J. H. Braslavsky, R. H. Middleton, and J. S. Freudenberg, "Feedback stabilisation over signal-to-noise ratio constrained channels," in Proc. 2004 Amer. Control Conf., Boston, MA, 2004, vol. 6, pp. 4903-4908.

[19] R. H. Middleton, J. H. Braslavsky, and J. S. Freudenberg, "Stabilization of non-minimum phase plants over signal-to-noise ratio constrained channels," in Proc. 5th Asian Control Conf., Melbourne, Australia, Jul. 2004, pp. 1924-1932.

[20] K. J. Åström, Introduction to Stochastic Control Theory. New York: Academic, 1970.

[21] G. N. Nair, R. J. Evans, I. M. Y. Mareels, and W. Moran, "Topological feedback entropy and nonlinear stabilization," TAC, vol. 49, no. 9, pp. 1585-1597, Sep. 2004.

[22] A. Sahai and S. Mitter, "The necessity and sufficiency of anytime capacity for stabilization of a linear system over a noisy communication link-Part I: Scalar systems," IEEE Trans. Inf. Theory, vol. 52, no. 8, pp. 3369-3395, Aug. 2006.

[23] R. H. Middleton and G. C. Goodwin, Digital Control and Estimation: A Unified Approach. : Prentice Hall, 1990.

[24] K. J. Åström and B. Wittenmark, Computer-Controlled Systems, 3rd ed. Englewood Cliffs, NJ: Prentice-Hall Int., 1997.

[25] N. Elia, "When Bode meets Shannon: Control-oriented feedback communication schemes," IEEE Trans. Autom. Control, vol. 49, no. 9, pp. 1477-1488, Sep. 2004.

[26] P. T. Kabamba, "Control of linear systems using generalized sampleddata hold functions," IEEE Trans. Autom. Control, pp. 772-783, Sep. 1987.

[27] G. F. Franklin, J. D. Powell, and M. Workman, Digital Control of Dynamic Systems, 3rd ed. Reading, MA: Addison-Wesley, 1998.

[28] N. Levinson and R. M. Redheffer, Complex Variables. New York: Holden Day, 1970.

[29] J. S. Freudenberg, R. H. Middleton, and J. H. Braslavsky, "Inherent design limitations for linear sampled-data feedback systems," Int. J. Contr., vol. 61, no. 6, pp. 1387-1421, 1995.

[30] J. S. Freudenberg, R. H. Middleton, and J. H. Braslavsky, "Robustness of zero shifting via generalized sampled-data hold functions," IEEE Trans. Autom. Control, vol. 42, no. 12, pp. 1681-1692, Dec. 1997.

[31] R. H. Middleton and J. S. Freudenberg, "Non-pathological sampling for generalized sampled-data hold functions," Automatica, vol. 31, no. 2, pp. 315-319, 1995.

[32] J. H. Braslavsky, M. M. Seron, D. Q. Mayne, and P. V. Kokotović, "Limiting performance of optimal linear filters," Automatica, vol. 35, no. 2, pp. 189-199, Feb. 1999.

[33] H. Kwakernaak and R. Sivan, Linear Optimal Control Systems. New York: Wiley Intersci., 1972.

[34] J. Chen, L. Qiu, and O. Toker, "Limitations on maximal tracking accuracy," IEEE Trans. Autom. Control, vol. 45, no. 2, pp. 326-331, February 2000.

[35] B. A. Francis, A Course in $H_{\infty}$ Control Theory. New York: SpringerVerlag, 1987.
[36] J. B. Conway, Functions of One Complex Variable, 2nd ed. New York: Springer-Verlag, 1978.

[37] M. Vidyasagar, Control System Synthesis: A Factorization Approach. Cambridge, MA: MIT Press, 1985.

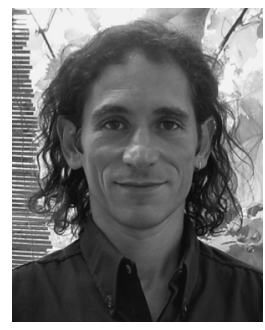

Julio H. Braslavsky (M’00) was born in Resistencia, Argentina, in 1962. He received the Ingeniero Electrónico degree from the National University of Rosario, Argentina, in 1989, and the Ph.D. degree from the University of Newcastle, Australia, in 1996.

He has held research positions with the University of Newcastle, the Catholic University of Louvain, Belgium, and the University of California, Santa Barbara. From 1999 to 2002, he was Adjoint Investigator with CONICET and Associate Professor with the National University of Quilmes, Argentina. Since 2002, Braslavsky is a Research Academic at the ARC Centre for Complex Dynamic Systems and Control at the University of Newcastle.

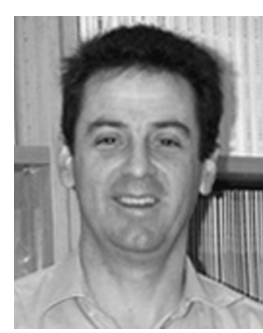

Richard H. Middleton (S'84-M'86-SM'94-F'99) was born in 1961 in Newcastle, Australia. He received the Ph.D. degree in 1987 from the University of Newcastle.

$\mathrm{He}$ is currently a Professor with the School of Electrical Engineering and Computer Science, University of Newcastle, and Director of the ARC Centre for Complex Dynamic Systems and Control. $\mathrm{He}$ is a Fellow of IEEE, has served as an associate editor of the IEEE TRANSACTIONS ON AUTOMATIC CONTROL, AND ON CONTROL SYSTEM TECHNOLOGY; and Automatica, and is currently Vice President (Conference Activities) of the IEEE Control System Society. His research interests include a broad range of Control Systems Theory and Applications.

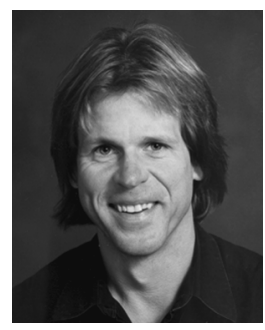

James S. Freudenberg (S'80-M'84-SM'97-F'00) was born in 1956, and grew up near Snake Run, IA. $\mathrm{He}$ received the B.S. degrees in mathematics and physics in 1978, and the M.S. and Ph.D. degrees in electrical engineering from the University of Illinois, Urbana, in 1982 and 1984, respectively.

$\mathrm{He}$ worked for a year with Rockwell/Collins Avionics. Since 1984, he has been on the faculty of the Electrical Engineering and Computer Science Department, University of Michigan, Ann Arbor, where he is a Full Professor. His interests are in the theory of fundamental limitations and the teaching of embedded control systems. 